\title{
Research and Application of Automated Testing Tool Based on STAF
}

\author{
Yu Shang ${ }^{1}$, XiaoLi Zhang ${ }^{2}$, YanBin Feng ${ }^{1}$, Momo Li ${ }^{1 *}$ \\ 1 School of Mathematics and Computer Science \\ Yunnan Nationalities University \\ Kunming 650031, P.R.China \\ 2 Faculty of Information Engineering and Automation \\ Kunming University of Science and Technology \\ Kunming 650031, P.R.China \\ *Corresponding author: E-mail: limomo99@163.com
}

\begin{abstract}
To improve the efficiency of automated testing, reduce testing spending, This article brings out a new automation testing framework-WTAF, and automated regression testing is the focus of the study. First of all, we choose the open source automation framework - STAF for regression testing, And then utilize STAF services to link all testing process implemented during the regression testing. Using automation testing technology, WTAF can successfully solve the low efficiency problem existed in manual or semi-automation testing.
\end{abstract}

Keywords-Automated testing; Regression testing; STAF; STAX.

\section{INTRODUCTION}

With the modern information technology development, the software system becomes more complex, which enhances the request for the function and performance of software, at the same time, the time spent on developing new edition of software is shortened ${ }^{[1]}$. In that case, how to guarantee the software quality becomes the key point which the enterprise should pay attention to. But in the traditional regression testing of many enterprises, people will usually use the semi-automation testing tools, which is very complicated for the configuration and the maintenance combined with more human intervention, and which will make the consumption of resources and time very large ${ }^{[2]}$. The long development cycle and lack of stability and efficiency of the testing tool also make obtaining high software quality difficult. In view of the actual need of the enterprise software product testing, this paper constructs a distributed, automated testing tool model - WTAF based on open source automation testing framework-STAF.

\section{RELATED CONCEPT}

\section{A. Regression testing}

In the process of the software development process, the major part of the testing work is regression testing[3]. The primary aim of regression testing is to confirm whether the functions which the system has before it is changed will be affected or not, in order to guarantee accuracy of the current changed function. In the gradual and the faster iteration development, That the new edition is issued continuously causes the frequent regression testing, but in the time which the product will be issued soon, the regression testing will be done several times every day. In order to confirm the correctness and the influence of the revision we require carrying on the regression testing.

When executing regression testing, firstly we should consider the bigger overlay scope and the less testing time, then we should carry on regression testing as automatic as possible.

\section{B. Open Source Testing framework - STAF}

Among the existed testing framework of the distributed software systems, STAF(Software Test Automation Framework)is developed and contributed to SourceForge(an open source community) by the IBM, which is a perfect choice in solving the distributed software system testing. STAF supports many hardware and software platforms, which is equal to the middleware of the testing tool in the function and the operational performance ${ }^{[4]}$.

STAF has the following characteristics:

- STAF operates in a peer-to-peer environment; in other words, there is no client-server hierarchy among running machine ${ }^{[5]}$.

- STAF is an Open Source automation framework designed around the idea of reusable components which is called services.

- It is intended to make it easier to create automated test case and workloads.

- STAF can help you increase the efficiency, productivity, and quality of your testing by improving your level of automation and reuse in your individual test case as well as your overall test environment. 


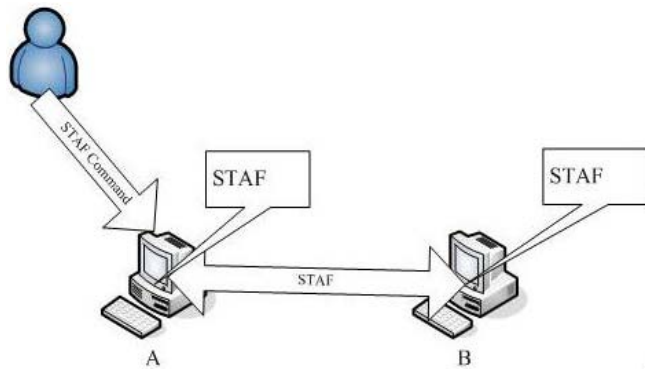

Figure 1. STAF principle diagram

\section{STAF execution engine - STAX}

STAX is an XML-based execution engine which is implemented as an external Java STAF service ${ }^{[6]}$. STAX is designed to make it significantly easier to automate the workflow of testing and test environments. STAX provides the following functions: supports the parallel running, custom operating control granularity, nested test case, controlling runtime, and supports existing Java and the Python module and so on. STAX has also provides a graphical monitor application, which makes you clearly to observe the place, the status and the error information of the running test case and so on .

\section{THE WTAF DESIGNING}

\section{A. The Selection of framework}

STAF has plenty of foundational services, flexible extensibility and excellent cross-platform performance, which can meet the requirement of the regression testing, also is the preferred platform to develop a testing tool.

\section{B. The WTAF module designing}

Generally speaking, the WTAF testing tool is mainly composed of the below five big function modules, its system architecture is presented as shown "Fig. 2":

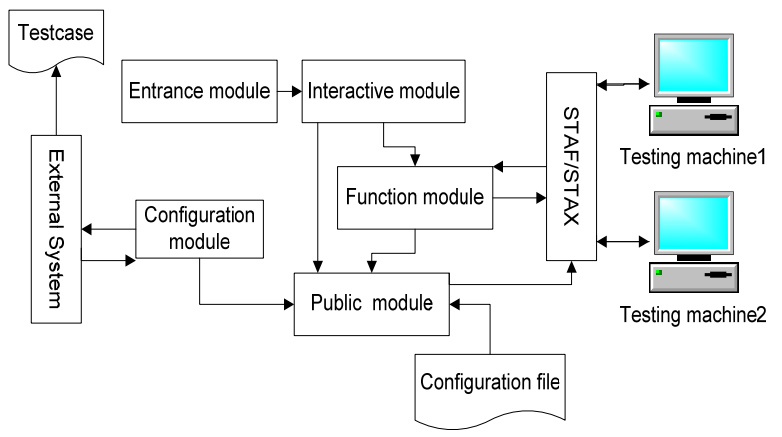

Figure 2. WTAF system Architecture

\section{1) configuration module}

In the process of testing when using WTAF tool, the needed test environment is configurable, it means testers firstly write these data in the corresponding configuration files, then testers can make a choice according to their own test scenario when executing testing. This configuration module includes needed information configuration about the user configuration, machine resource configuration as well as relevant software integration. During the process when testing is executed, the testing tool can retrieve the needed data from different test scenes, with which substitutes the variables of the test drivers and executing scripts.

2) Entrance module

To start the WTAF testing tool through graphical interface or command line, we must begin with the Main file. Because Main file is the entrance of WTAF testing tool, also is the core part of the overall tool, every job will begin with this function. The main tasks: calling public module WTAFMain; executing some necessary test operations; also defines CaseMain function about every test function point, which mainly set some variables of test suite as well as select running mode and so on. The function of the entrance module is more stable and simpler in structure than other modules. On the whole, its most major characteristic is a test procedure driven by WTAF really, which is different from general automated testing tool driven through specific test program. Thus this method guarantees WTAF integrity, and own the real monitor privilege with the entire test flow.

3) Public module (WTAFMain)

Public module is the fundamental framework of the automation testing tool -WTAF, in which implementation logic relative to the test case is represented. Although for different test case, the test scenes and the test function points are not the same, The entrance module Main function after having handle the corresponding parameters firstly call WTAFMain function of this module .The main tasks are as follows:

- Clean the last test environment to wipe off the remainder of the last testing, to guarantee the correctness of the testing result. For example delete the last test directory, resume to the initial state .

- Examine the variable validity, including WTAF variable, programmer-defined variable.

- Set all the variables needed during the test process: including WTAF global variable, Server variable, Client variable, local variable and so on.

- Call the logical function CaseMain of the entrance module, which carries out the corresponding operation.

- Deal with the test result, record the failing test case, store the related log file.

- After completing the test, create test result document, and send it to the server preserving test result.

- require exceptional control during the entire test process.

That means all regression Test case will be run by calling this module.

4) Ffunction module (WTAFFunction)

This module has encapsulated all common STAF services. API (Application Program Interface) of one program means the collection of some correlation orders, the function and the communication protocol[7]. Once testers have these APIs, they can build automation testing 
tool utilizing them, create the test automation procedure, which save time and manpower. WTAF function module is composed of these API sets, which is the core of the automation testing tool.

So how to design these API, we need to know STAF service. STAF adopts the internal service and external service. Internal STAF service Provides the core services in STAFProc, for instance data management and synchronization etc, which can be reusable, then in the basis of which other services can be created. External STAF services are dynamically started by STAF through the sharing library, these External services can make it easier to communicate with the outside, for instance the log service, which is an external service, can help user to record and to look for these logs.

The following is illustrated according the designing of the log analysis API: We know any test work will produce a large number of logs, for instance the setup log and the test result $\log$ and so on, and the log may be distributed in the testing machine, whose place and operating system are both different. Testers rush about among every different machine in order to analyze these log, Even remote operation can be used, testers have to grasp many kinds of operating systems and register the multi-machines in order to carry on the analysis, Moreover many log files are verbose, which make testing work complicated and dull. Therefore, we need to design a $\log$ analysis service to solve this problem .This service flow chart is presented as shown in "Fig. 3":

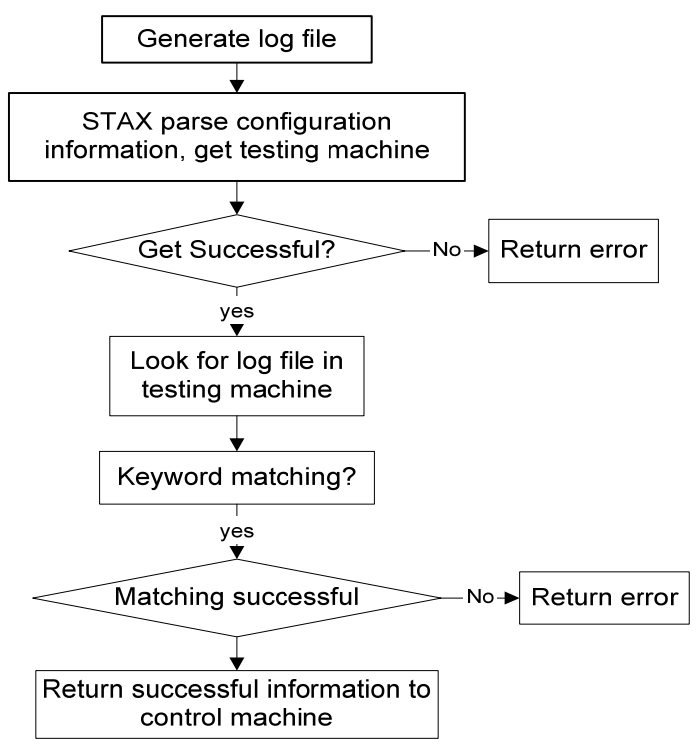

Figure 3. Log analysis service flowchart

Shown from the log analysis service flow chart, The test logic is implemented by testers according to the actual test request, but the log service provided by STAF is merely the lowest level calling, it does not contain the preparatory work for the test related products and the multi-match keyword information in the process and so on. Therefore, the realization of these API are extremely important. This module is also the core part of the WTAF testing tool.
5) interactive modules

In this project, we will utilize the two software: CVS and ARTTS. They are edition control and result analysis software, So we will finish the integration of WTAF and CVS as well as WTAF and ARTTS in this module. we will finish it by compiling the corresponding function in the configuration module.

\section{Wokflow of WTAF}

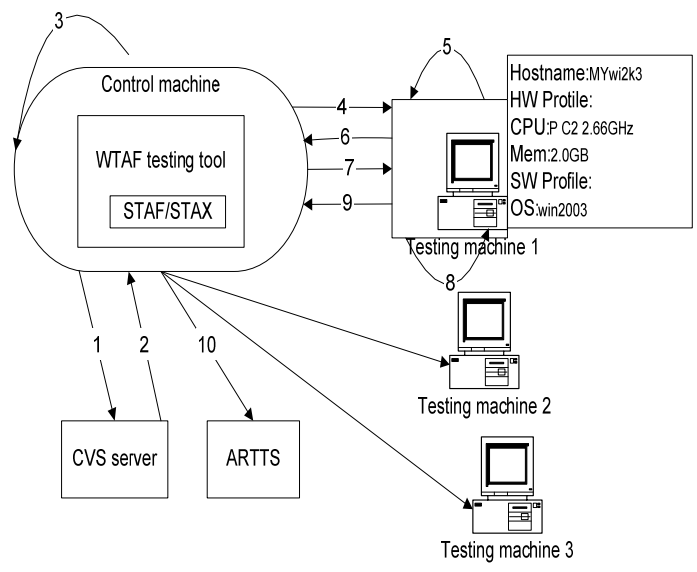

Figure 4. workflow of WTAF

The basic flow of the automated testing tool is started as follows, exclusive of the environment clean-up work of the start and the end :

- Send updating testing code request To CVS server.

- Get corresponding newest testing code According to demand, and guarantee consistency of testing code.

- Analyze and look for the corresponding testing machine according to the configuration information of the configuration module.

- Send the testing code to testing machine.

- Compile the testing code automatically( carry on different compile on different target machine), if make a mistake, terminate the test automatically, and retain the scene

- Return result information.

- Send related command to execute test logic, including creating the resource pool, producing configuration files such as ubb, dubb according to the test case description, compile code, execute the calling command and so on.

- Run script according the corresponding command, execute specific test logic.

- Automatically Diagnose testing whether is successful or not and return results.

- Demonstrate the test result and automatically send it to the designated server.

\section{SUMMARY}

By utilizing the basic services provided by STAF, WTAF can realize automated downloading and the 
transmission of the test code, graphical display of the test condition and auto-generation of the test report, which can avoid the resources conflict, enhance the reusability. Using the STAX execution engine to call the services provided by STAF, we can realize automated compiling of the test cases and automated configuring of the test environment, improve the efficiency and the quality of software, meet the tester's need. Testers finish the automated testing by compiling XML scripts to call STAF services via STAX, which improve the generality, reduce the study time, and make WTAF simple and easy to use actually.

\section{REFERENCES}

[1] Patton R, “ software testing,” [M ]. Beiijing, China Machine Press, 2002.
[2] Subram anyan Rajesh, “Test Automation in Practice," [C]. 31stAnnual International on Computer Software and Applications Conference, 2007, COM PSAC, 2007(1): 9- 19.

[3] Daniel J Mosley,Bruce A Posey, “Just Enough Software Test Automation,” Indianapolis,Prentice Hall PTR, 2002

[4] David Bender., “Getting Started with STAF,” [ EB /OL]. May 2008.

[5] STAF V3 User's Guide, IBM Inc. http://staf.sourceforge.net/ current/STAFUG.htm.

[6] STAX Services User's Guide, IBN Inc. http://staf.sourceforge.net/ current/staxug.pdf.

[7] Kanglin Li, Mengqi Wu, "Effective Software Test Automation," Berkeley SYBEX, 2004. 